Thélème. Revista Complutense de Estudios Franceses

ISSN-e: $1989-8193$

http://dx.doi.org/10.5209/THEL.57938

\title{
Analyse linguistique des données chiffrées dans les textes de vulgarisation scientifique
}

\author{
Riham El Khamissy ${ }^{1}$
}

Recibido: 09 de septiembre de 2017 / Aceptado: 09 de noviembre 2017

Résumé. Dans le présent article, notre objectif consiste à saisir comment les journalistes traitent l'information de nature quantitative, notamment lorsqu'il s'agit de nombres. Nous avons choisi de travailler sur les articles de vulgarisation scientifique parce qu'ils foisonnent en chiffres. Les textes sélectionnés parlent du virus Zika, virus qui connait une expansion planétaire importante aux cours des années 20152016. Nous explorerons d'abord le niveau formel des données chiffrées et leur intégration dans le texte. Ensuite, nous examinerons à fond le co-texte des chiffres, autrement dit les éléments qui permettent de modifier les données chiffrées et/ou de (ré)-orienter l'interprétation.

Mots-clés: données chiffrées; quantifieurs; approximation; opérateurs argumentatifs; Zika

\section{[en] Linguistic analysis of figures in articles of scientific popularisation}

\begin{abstract}
In this paper, our goal is to understand how journalists deal with quantitative information, especially when it comes to numbers and figures. We have chosen to work on articles of scientific popularisation because they display abundant figures. The selected texts speak of Zika virus, which has had an important expansion during the last two years (2015-2016). We shall first explore the formal level: numbers and their integration into the text. Subsequently, we shall examine in detail the co-text of the numbers, in other words the elements that make it possible to modify the figures and / or (re)-orient their interpretation.
\end{abstract}

Keywords: Numbers; quantifiers; approximation; argumentative operators; Zika

\section{[es] Análisis lingüístico de números en artículos de vulgarización científica.}

Resumen. En este artículo, nuestro objetivo es comprender cómo los periodistas manejan la información cuantitativa, especialmente cuando se trata de números. Elegimos trabajar en artículos científicos populares porque abundan en números. Los textos seleccionados hablan del virus Zika, un virus que está experimentando una expansión global significativa en los años 2015-2016. Primero exploraremos el nivel formal de datos encriptados y su integración en el texto. Luego, examinaremos a fondo el co-texto de las figuras, es decir, los elementos que hacen posible modificar las figuras y / o (re) orientar la interpretación.

Palabras clave: datos numéricos; cuantificadores; aproximación; operadores argumentativos; Zika.

Cómo citar: Khamissy, R.E. (2018) «Analyse linguistique des données chiffrées dans les textes de vulgarisation scientifique » Thélème. Revista Complutense de Estudios Franceses, Vol. 33, Núm. 1 : 29-44.

Faculté des langues (AL ALSUN). Université Ain Shams.

Email: rihamelkhamissy@yahoo.fr 
Sommaire: 1. Introduction. 2. Recueil des données, filtrage et échantillonnage. 3. Analyses. 3.1. En chiffres ou en lettres ? 3.2. Les chiffres et leur co-texte. 3.2.1. Les marqueurs d'approximation. 3.2.2. Les opérateurs argumentatifs. 3.3. Le tout et le néant. 3.4. Les chiffres exacts : vérité ou illusion ? 4. Conclusion

\section{Introduction}

On dit généralement que les chiffres (dénombrement, score, sondage, statistique, pourcentage, etc.) sont parlants autant que les mots, et parfois même davantage.

En langue, l'adjectif numéral (désormais Num) se trouve rarement étudié en dehors de la grammaire traditionnelle normative. Cette grammaire lui consacre quelques paragraphes qui abordent son orthographe, sa forme (simple ou composée), son caractère invariable (pour la majorité des adjectifs numéraux cardinaux), ses éventuels accords (lorsqu'il s'agit de vingt et de cent).

Dans la presse écrite, les journalistes peuvent rapporter une statistique de sorte que celle-ci devienne l'élément central de l'article (chiffrage de l'information), l'explication des chiffres ne constituant que l'information secondaire. Or, le plus souvent, les statistiques et les pourcentages servent à appuyer le texte même. Le cas échéant, la quantification devient « un moyen de donner une dimension supplémentaire aux discours préexistants en les renforçant par des données chiffrées. » (Vanneuville, $2014:$ 126).

Or, une interrogation s'impose et se trouve à l'origine de notre réflexion : est-ce la mise en chiffres qui accrédite la véracité du discours ou est-ce le discours qui altère les données en chiffres?

Notre travail a pour objectif de saisir comment les journalistes traitent l'information chiffrée dans les médias de vulgarisation ${ }^{2}$ et dans la presse généraliste, et ce autour d'un thème, celui du virus Zika, virus qui connait une expansion importante aux cours des deux dernières années (2015-2016). Les articles traitant ce thème regorgent de données quantitatives, et notamment de chiffres. Nous avons opté pour les textes de vulgarisation plutôt que les textes scientifiques parce que l'une des finalités de notre travail consiste à mettre en relief la volonté d'orienter le destinataire vers une attitude ou une conclusion donnée. À notre sens, ce phénomène se manifeste davantage dans les textes de vulgarisation destinés au grand public, généralement non averti.

Notre contribution s'inscrit dans la même lignée que les travaux d'Adler et Asnès $(2004,2007,2013)$ ainsi que les travaux sur l'orientation argumentative développés par Ducrot $(1983,1995,2002)$ et repris par Doury et Moirand (2004).

La question que nous traitons, dans le présent article, n'est pas le recours aux chiffres en soi mais plutôt leur mise en discours et leur soumission aux objectifs des journalistes pour influencer l'opinion publique.

En 1988, Jacobi et Schiele parlent de la reformulation comme paramètre linguistique des textes de vulgarisation scientifiques. En 2009, Hyland identifie, dans son ouvrage anglais, les caractéristiques des textes de vulgarisation scientifique comme suit : utilisation des explications, des dispositifs cohésifs tels que la répétition, l'emploi de la synonymie ou les articles démonstratifs, l'implication fréquente du destinataire souvent interpellé par les marques de la deuxième personne you, your. (En français, il s'agit de la deuxième personne du pluriel ex. « vous », « votre », « vos »). 


\section{Recueil des données, filtrage et échantillonnage}

Définir un corpus oblige à « circonscrire un objet clos et donc à se donner des règles pour garantir un minimum de représentativité à cet objet, cette représentativité étant elle-même liée à un objectif d'étude. » (Condamines et Dehaut, 2011 : 266).

La recherche par le mot-clé «Zika » a donné comme résultat 13090 documents en français depuis deux ans (1er janvier 2015 - 31 décembre 2016) sur la base de données médiatiques « Europresse.com ». Après avoir filtré ces documents par élimination des doublons, nous avons tenu à écarter manuellement quelques articles répertoriés qui ne sont pas pertinents : les articles où «Zika» est un Nom Propre de personne et ceux qui ne contiennent pas de données chiffrées.

Étant donné que nous cernerons de près uniquement le dénombrement par chiffrage $^{3}$, autrement dit les chiffres qui quantifient ou qui classifient, seront donc exclus de notre champ d'investigation les dates, les indications de l'heure, les numérotations en listes et celles qui renvoient à des références (notes de fin de textes ou notes infra-paginales), les chiffres dans les adresses postales ou dans les Url et liens renvoyant à des sites Internet.

Notre corpus étant homogène ${ }^{4}$, nous avons déterminé un échantillon représentatif à partir duquel nous analyserons les données chiffrées en profondeur. Nous avons calculé la taille de l'échantillon représentatif avec le calculateur d'échantillon sur le site https://fr.checkmarket.com/calculateur-taille-echantillon/ qui opère selon la formule de statistique de Krejcie et Morgan ${ }^{5}$ avec marge d'erreur de $5 \%{ }^{6}$ et niveau de confiance $95 \%$. Ainsi, notre échantillon de travail comptabilise 374 articles de presse, au total 65322 mots.

Les articles sont extraits des journaux, des revues et de la presse sur le web mentionnés ci-dessous :

\begin{tabular}{|c|c|c|c|}
\hline Nom de la source & Type & Périodicité & $\begin{array}{c}\text { Couverture géogra- } \\
\text { phique }\end{array}$ \\
\hline L'Observateur.fr & Presse web & quotidien & internationale \\
\hline Le Monde.fr & Presse web & quotidien & internationale \\
\hline La Matinale du Monde & Presse web & quotidien & internationale \\
\hline
\end{tabular}

\footnotetext{
Linguistiquement parlant, Olivier Soutet (2010 : 22) reconnait deux types de déterminants servant au dénombrement : « déterminants du dénombrement par chiffrage (ex. adjectifs numéraux cardinaux) et déterminants du dénombrement sans chiffrage (il s'agit de déterminants de la quantification ordonnés sur l'échelle conduisant de ne aucun à tous les) ou encore un article.

4 Tous les textes recueillis appartiennent au même domaine, à savoir à la vulgarisation scientifique, et traitent le même thème

$5 \quad \mathrm{SS}=(\mathrm{Z} \text {-score })^{2} * \mathrm{p} *(1-\mathrm{p}) /(\text { margin of error })^{2}$ pour populations infinies et $\mathrm{SS}$ adj $=(\mathrm{SS}) / 1+[(\mathrm{SS}-1) / \mathrm{popu}-$ lation] pour population finies

$\mathrm{SS}=$ sample size, $\mathrm{P}=$ proportion (prenez 0,50 comme estimation si inconnu) $)$ https://fr.checkmarket.com/ calculateur-taille-echantillon/

6 «C'est le nombre positif ou négatif qui est généralement affiché dans des résultats des sondages d'opinion de journaux ou de télévision. Par exemple, si vous utilisez une marge d'erreur de $4 \%$ et $47 \%$ de votre échantillon donne une certaine réponse, vous pouvez en être «sûr» que si vous auriez posez cette question à la population entière, entre $43 \%(47-4)$ et $51 \%(47+4)$ auraient donné la même réponse (un intervalle d'erreur moins grand demande une taille d'échantillon plus grande). » source : https://fr.checkmarket.com/calculateur-taille-echantillon/
} 


\begin{tabular}{|c|c|c|c|}
\hline Libération & Presse Web & quotidien & nationale (France) \\
\hline Libération.fr & Presse web & quotidien & nationale (France) \\
\hline L'Express.fr & Presse web & quotidien & nationale (France) \\
\hline Le Point.fr & Presse Web & En continu & nationale (France) \\
\hline La lettre de l'Expansion & $\begin{array}{c}\text { Journal impri- } \\
\text { mé }\end{array}$ & hebdomadaire & nationale (France) \\
\hline La Croix & $\begin{array}{c}\text { Journal } \\
\text { imprimé } \\
\end{array}$ & quotidien & $\begin{array}{c}\text { nationale } \\
\text { (France) }\end{array}$ \\
\hline La Tribune & $\begin{array}{l}\text { Journal } \\
\text { imprimé }\end{array}$ & quotidien & nationale (France) \\
\hline La République.fr & Presse Web & quotidien & $\begin{array}{c}\text { régionale } \\
\text { (Pau, Aquitaine, } \\
\text { France) }\end{array}$ \\
\hline Le Figaro & $\begin{array}{l}\text { Journal } \\
\text { imprimé }\end{array}$ & quotidien & nationale (France) \\
\hline Le Figaro.fr & $\begin{array}{l}\text { Presse } \\
\text { web }\end{array}$ & quotidien & $\begin{array}{c}\text { nationale } \\
\text { (France) }\end{array}$ \\
\hline Challenges & Presse Web & quotidien & nationale (France) \\
\hline Marianne & $\begin{array}{l}\text { Revue } \\
\text { imprimée }\end{array}$ & Hebdomadaire & nationale (France) \\
\hline Le Particulier.fr & Presse web & En continu & $\begin{array}{l}\text { nationale } \\
\text { (France) }\end{array}$ \\
\hline RiskAssur & Presse web & Hebdomadaire & $\begin{array}{c}\text { nationale } \\
\text { (France) }\end{array}$ \\
\hline Sud Ouest & $\begin{array}{l}\text { Journal } \\
\text { imprimé }\end{array}$ & quotidien & $\begin{array}{l}\text { Régionale (Lot et } \\
\text { Garonne, France) }\end{array}$ \\
\hline Le Parisien & $\begin{array}{l}\text { Journal } \\
\text { imprimé }\end{array}$ & quotidien & $\begin{array}{l}\text { régionale (Paris, } \\
\text { France) }\end{array}$ \\
\hline 20minutes.fr & Presse web & quotidien & $\begin{array}{c}\text { nationale } \\
\text { (France) }\end{array}$ \\
\hline L'Est Républicain & $\begin{array}{l}\text { Journal } \\
\text { imprimé }\end{array}$ & quotidien & $\begin{array}{c}\text { régionale (Lorraine, } \\
\text { France) }\end{array}$ \\
\hline Atlantico & Presse web & quotidien & nationale (France) \\
\hline Science et Avenir & Presse Web & quotidien & nationale (France) \\
\hline Ouest France.fr & Presse Web & quotidien & $\begin{array}{c}\text { régionale (Bretagne, } \\
\text { France) }\end{array}$ \\
\hline Midi Libre.fr & Presse Web & quotidien & $\begin{array}{c}\text { régionale (Langue- } \\
\text { doc-Rousillon, France) }\end{array}$ \\
\hline Nord Éclair & Presse Web & quotidien & $\begin{array}{c}\text { Régionale } \\
\text { (Roubaix, Nord-Pas- } \\
\text { De-Calais, France) }\end{array}$ \\
\hline L'Usine Nouvelle.com & Presse Web & quotidien & nationale (France) \\
\hline Les Echos.fr & Presse Web & En continu & nationale (France) \\
\hline
\end{tabular}




\begin{tabular}{|c|c|c|c|}
\hline Le Quotidien du Médecin & $\begin{array}{l}\text { Journal } \\
\text { imprimé }\end{array}$ & $\begin{array}{l}\text { bihebdomadaire } \\
\text { ou } \\
\text { trihebdomadaire }\end{array}$ & nationale (France) \\
\hline $\begin{array}{l}\text { Journal de l'environne- } \\
\text { ment.com }\end{array}$ & Presse Web & quotidien & $\begin{array}{l}\text { nationale } \\
\text { (France) }\end{array}$ \\
\hline Aujourd'hui en France & Presse Web & En continu & nationale (France) \\
\hline Le Nouvelliste & $\begin{array}{l}\text { Journal } \\
\text { imprimé }\end{array}$ & quotidien & $\begin{array}{c}\text { régionale } \\
\text { (Trois-Rivières, Qué- } \\
\text { bec, Canada) }\end{array}$ \\
\hline Le Quotidien & $\begin{array}{l}\text { Journal } \\
\text { imprimé }\end{array}$ & quotidien & $\begin{array}{c}\text { régionale } \\
\text { (Québec, Canada) }\end{array}$ \\
\hline $\begin{array}{l}\text { Le Journal du dimanche } \\
\text { (JDD) }\end{array}$ & Presse web & hebdomadaire & $\begin{array}{c}\text { nationale } \\
\text { (France) }\end{array}$ \\
\hline $\begin{array}{l}\text { CNWTELBEC } \\
\text { Canada Newswire }\end{array}$ & $\begin{array}{l}\text { Communiqué- } \\
\text { de Presse }\end{array}$ & En continu & $\begin{array}{c}\text { Régionale } \\
\text { (Montréal, Québec, } \\
\text { Canada) }\end{array}$ \\
\hline L'Agefi hebdo & $\begin{array}{l}\text { Journal } \\
\text { imprimé }\end{array}$ & hebdomadaire & nationale (France) \\
\hline RFI.fr & $\begin{array}{l}\text { Presse web de } \\
\text { Radio France } \\
\text { internationale }\end{array}$ & En continu & internationale \\
\hline
\end{tabular}

\section{Analyses}

Les nombres se rencontrent à l'écrit en chiffres (arabes ou romains) ou en toutes lettres (un, deux, trois etc.). Au niveau formel, nous explorerons les motifs du choix entre la forme typique et classique du nombre (un nombre s'écrit en principe en chiffres) et sa transcription en lettres graphiques (le recours à l'adjectif numéral). Ensuite, nous cernerons de près les données chiffrées dans leur environnement textuel.

\subsection{En chiffres ou en lettres?}

L'écriture en chiffres a un atout indéniable : la saillance visuelle dans l'espace textuel.

Dans notre échantillon, les nombres écrits en chiffres recensent les taux d'infection, la vitesse de la contagion ou de la propagation de la maladie, le nombre de pays touchés par le virus Zika :

(1) Le frelon asiatique, arrivé accidentellement de Chine via les caisses transportant les poteries il y a onze ans, a déjà colonisé $\mathbf{7 0} \%$ de notre territoire. (L'Est Républicain, 16/8/2015)

(2) Au total, 22 zones sont désormais concernées par ce dispositif de lutte contre ce risque de dissémination de la dengue et du chikungunya mais également du virus Zika. (Le Particulier.fr, 7/9/2015) 
(3) En 2014, en France métropolitaine, $\mathbf{4 8 9}$ cas de chikunguya et de 201 cas de dengue ont été déclarés. (Le Particulier.fr, 7/9/2015)

(4) Au Brésil, où le Zika est très présent, les autorités évoquent $\mathbf{3 0 0 0}$ cas de microcéphalie ${ }^{7}$ depuis 2015. (Les Échos, 30/1/2016)

Dans les exemples susmentionnés, nous constatons que non seulement le nombre écrit en chiffres est facilement repérable dans le texte mais il fait aussi souvent le point sur l'ampleur de la menace : les chiffres sont alarmants et créent une onde-choc chez le destinataire, allant au-delà de la sensibilisation pour maximiser le danger.

Par ailleurs, nous avons remarqué que les chiffres transcrits en lettres, dissimulés dans la texture des écrits de vulgarisation, sont généralement ceux qui ont l'effet d'atténuation. Les journalistes, en optant pour les formes simples plutôt que les formes composées, visent à dédramatiser et à positiver les faits :

(5) Trois décès liés au virus Zika confirmés en Colombie. (Titre, Le Figaro. fr, 5/2/2016)

(6) Depuis 2015, 70 pays ont signalé la présence du virus, dont 55 au stade épidémique et cinq où la transmissions du virus est permanente (Le Monde.fr, 21/9/2016)

(7) Sur neuf mois d'épidémie, les médecins estiment à près de 10000 patients consultés pour une contamination Zika (Aujourd'hui en France, 21/10/2016)

Lorsque le nombre (n) est grand ( $\mathrm{n}>20)$, la tendance est de le garder tel quel, alors que lorsqu'il est petit $(\mathrm{n} \leq 20)$, il est transcrit en lettres.

Les chiffres écrits en lettres figurent souvent dans les titres des articles de presse : ils montrent que, quoique facilement transmissible, le virus responsable de cette maladie a souvent des conséquences bénignes, rarement malignes:

(8) Le virus Zika en cinq questions. (Le Figaro, 22/1/2016)

(9) Zika : dix questions sur un virus qui inquiète (Le Monde.fr, 2/2/2016)

Bien que les questions posées en (8) et (9) pour comprendre le virus soient peu nombreuses (cinq et dix), elles sont suffisantes pour connaître le risque émergent et pour s'en prévenir.

La formulation en lettres des données chiffrées leur ôte une part de leur austérité en activant le topos du " peu » et du " petit», donc de la quantité faible et négligeable (ex. 10 et 11). Ceci permet de minimiser le danger, sans pour autant le bannir complètement.

(10) Le virus Zika devrait disparaître de lui-même d'ici à trois ans. (Titre, Aujourd'hui en France, 14/7/2016)

Maladie de déformation cérébrale chez les fœtus et les bébés à naître. Le virus Zika est fortement suspecté d’être étroitement lié à cette anomalie.

8 Les maladies liées au virus Zika sont la microcéphalie et le syndrome de Guillain-Barré. 
(11) L'épidémie actuelle de Zika devrait s'éteindre d'elle-même dans les trois ans ${ }^{9}$. (Sciences et Avenir, 18/7/2016)

À noter que, dans de rares occurrences, les chiffres non-transcrits sont à prendre dans une optique plutôt positive et rassurante, rejoignant ainsi les chiffres transcrits en toutes lettres : tel est le cas des données chiffrées qui renvoient aux investissements dans le secteur de la recherche en immunologie et en microbiologie pour endiguer l'épidémie. C'est ce qu'illustrent les exemples (12) et (13) où le chiffre, en dépit de sa saillance visuelle, n'effraie pas mais apporte, dans ce contexte d'épidémie, une lueur d'espoir :

(12) Chaque année, $\mathbf{2}$ milliards de dollars sont consacrés à la traque et à l'éradication. (Marianne, 11/12/2015)

(13) Le président américain Barack Obama a annoncé lundi 8 février son intention de débloquer $\mathbf{1 , 8}$ milliard de dollars pour la prévention et la recherche sur Zika (...) (Sciences et Avenir, 10/2/2016)

Dans de tels cas, c'est la subjectivité liée aux normes sociales qui détermine à partir de quel chiffre les dépenses en matière de santé publique reflètent l'importance accordée à ce secteur et réconforte les citoyens, et de ce fait, deviennent un atout.

Le Num ordinal, écrit en toutes lettres, est moins fréquent dans notre corpus que le Num cardinal et reflète une tendance à la comparaison et au classement. Selon l'analyseur de texte et de mot en ligne lexicool, notre échantillon présente la statistique suivante concernant le Num ordinal premier-e-s :

$\begin{array}{ccc}\text { Mots } & \text { Occurrences } & \text { Rang } \\ \text { Première(s) } & 7 & 21 \\ \text { Premier(s) } & 3 & 22\end{array}$

Cet ordinal marque généralement le début du danger et les premières alertes (14) et (15) ou l'historique de l'épidémie (16) :

(14) Le virus Zika est arrivé en Guyane et en Martinique, où sont identifiés deux premiers cas de personnes contaminées par cette nouvelle maladie émergente qui pourrait aussi prendre pied en métropole. (Le Figaro, 21/12/2015)

(15) La première publication faisant état d'une transmission du virus par le biais d'une relation sexuelle remonte à 2011. (Le Monde.fr, 21/9/2016)

(16) Après avoir provoqué une première épidémie en Micronésie en 2007, il s'est propagé en 2013-2014 en Polynésie, puis en début de cette année, au Brésil où sévissent les insectes tigres. (RiskAssur, 24/8/2015)

Parfois, le Num « première » apparaît en collocation avec le substantif « fois » / Num+fois/ :

«Le déterminant dans les + numéral ne relève pas en soi de la quantité faible (même si la substitution à quelques et le test de la négation vont dans ce sens) mais dénote une quantification non extensive : dire $J$ 'ai lu dans les trente romans peut paraître plus modeste, moins péremptoire, que J'ai lu trente romans ». (Vaguer, 2005 : 124) 
(17) Identifiée pour la première fois sur un macaque rhésus dans une forêt ougandaise qui lui a donné son nom, la fièvre Zika est pourtant restée négligée jusqu'aux épidémies micronésiennes et polynésiennes. ( $\mathrm{Le} \mathrm{Fi}$ garo, 21/12/2015).

(18) La surveillance de cette expansion territoriale est un souci constant car la présence de ce moustique en métropole, détectée pour la première fois en 2004, a introduit jusqu'ici un risque inexistant : la possibilité d'une épidémie de chikungunya et/ou de dengue, voire de Zika désormais en France métropolitaine. (Sciences et Avenir, 28/5/2015)

(19) Il (le Zika) a été identifié pour la première fois en Amérique du sud il y a moins de deux ans, mais il s'est rapidement répandu sur le continent. (L'Express, 30/12/2015)

(20) Une équipe franco-thaïlandaise vient d'identifier, pour la première fois comment le virus Zika, cousin du chikunguya et de la dengue, infecte les cellules humaines. (Sciences et Avenir, 2/7/2015)

(21) L'existence d'un lien entre Zika et la microcéphalie du fœtus a été établi scientifiquement pour la première fois dans une étude publiée le 4 mars dernier dans le revue scientifique Cell Stem. (Le Figaro.fr, 22/3/2016)

Dans les exemples, /première+fois/ vise soit la première apparition de la maladie (17 à 19) soit le caractère unique et exceptionnel du virus comme en témoignent les occurrences (20) et (21) où cet ordinal met l'accent sur la découverte scientifique d'un fait jusqu'alors ignoré.

\subsection{Les chiffres et leur co-texte}

Le chiffre est rarement indépendant de son entourage textuel. L'environnement linguistique immédiat des chiffres, communément désigné par le co-texte, peut modifier le chiffre en matière d'exactitude, de précision et/ou d'orientation argumentative, de valeur positive ou négative.

Sur ce, nous procédons à l'analyse de quelques quantifieurs, catégorisés par la grammaire en tant que locutions prépositives indiquant l'espace ou le temps et dont la valeur de quantification a été soulignée par certains chercheurs, citons entre autres, Melis (2003), Adler et Asnès (2004, 2007, 2008, 2010, 2013).

\subsubsection{Les marqueurs d'approximation}

Le patron /quantifieur $+\mathrm{Num} /$ comme /près de $+\mathrm{Num} /$, lenviron $+\mathrm{Num} /$, lautour + Num/, /près de + Num/, /plus de + Num/, /moins de + Num/, lau-delà de + Num/, / jusqu'à $+\mathrm{Num} /$ incarne la modification du numéral cardinal. Or, chaque quantifieur a un sémantisme qui affecte différemment le nombre qui le suit.

/Près de+Num/ est le marqueur d'approximation par rapprochement le plus fréquent dans notre échantillon représentatif :

Mot

près de
Occurrences
Rang

11 
Chaque fois qu'un chiffre est précédé de près de, « il y a une tentative de rapprochement vers le nombre donné sans toutefois pouvoir atteindre ce nombre » (Adler et Asnès, 2010 : 41). La séquence /près de + Num/ constitue un cas d'arrondissement $^{10}$ asymptotique ${ }^{11}$ exclusivement par excès ${ }^{12}$ et présente le nombre, immédiatement inférieur à la valeur donnée, comme égal à ou indissociable de cette valeur. Considérons les exemples suivants :

(22) Zika provoque peu de symptômes ou même ne provoque pas de symptômes dans près de $80 \%$ des cas. (La Croix, 22/12/2015)

(23) Près de $80 \%$ des personnes infectées ne présentent pas de symptômes, ce qui n'empêche pas de propager le virus en étant piquées par un moustique vecteur. (Le Figaro, 21/12/2015)

(24) Début mars, une étude prospective menée à Rio sur une cohorte de femmes enceintes a mis en évidence près de $30 \%$ d'anomalies fœtales lors d'infection Zika en cours de grossesse. (Le Quotidien du Médecin, 29/3/2016)

(25) Aux États-Unis, près de 2500 cas de Zika ont été recensés sur le continent dont 584 femmes enceintes. (Le Quotidien du Médecin, 29/8/2016)

Précédé du quantifieur près de, le chiffre n'est plus seulement informatif mais argumentatif. Il est censé solliciter l'attention du récepteur et l'orienter, argumentativement parlant, vers des conclusions du types /ce virus est dangereux/ : même si avec « près de $80 \%$ » en (24) et (25), le chiffre exact du pourcentage n' est pas atteint pour ce qui est de l'absence de symptômes patents, ni non plus atteint avec le « près de $30 \%$ » en (26) pour ce qui est de l'anomalie fœtale. Toutefois, la situation reste alarmante et l'approximation n'est pas, sur le plan de la conscientisation, moins efficace que le chiffre exact, puisqu'elle est orientée vers des conclusions identiques.

Le patron /jusqu'à $+N u m /$ produit un effet pragmatique analogue : le nombre mentionné et tous les nombres qui lui sont inférieurs sont des arguments co-orientés vers la même conclusion. La séquence contenant /jusqu'à $+N u m /$ se distingue de / près de $+N u m /$ par les points suivants : d'abord, dans /jusqu'à $+N u m /$, la limite supérieure indiquée par le chiffre donné est atteinte et reste « incluse dans le domaine dénotationnel $»^{13}$ (Adler et Asnès, 2013 : 17). Aux termes de ces deux linguistes, il est question d' « approximation par atteinte ». Par conséquent, l'accomplissement n'est pas discrédité. En outre, /jusqu'à $+N u m /$ est scalaire ${ }^{14}$ : il exclut tous les nombres supérieurs au point de référence et inclut toutes les unités inférieures ainsi que le chiffre pris comme point de référence. Or, il n'exclut pas les unités qui ne sont pas immédiatement inférieures au point culminant (le chiffre limite), comme le fait le patron /près de $+N u m /$. Tout comme /près de $+N u m /$, /jusqu'à $+N u m /$ tend à persuader les destinataires de la menace qui plane. Toutefois, il est peu fréquent dans notre échantillon :

\footnotetext{
Procédé connu en mathématique qui consiste à remplacer un nombre par sa valeur rapprochée.

« En ce sens que le nombre dans sa portée ne peut jamais être atteint ». (Adler et Asnès, 2008)

Vers une valeur supérieure.

Jusqu'à n'est donc pas asymptotique.

«Construit une échelle de nombres de sorte que certains y sont inclus d'autres en sont exclus ». (Adler et Asnès, $2010: 40)$
} 


$\begin{array}{ccc}\text { Mot } & \text { Occurrences } & \text { Rang } \\ \text { Jusqu'à } & 3 & 12\end{array}$

(26) Jusqu'à présent, des transmissions sexuelles du virus Zika ont été reportées jusqu'à $\mathbf{4 8}$ jours après le début des symptômes et la présence dans le sperme a été relevée jusqu'à $\mathbf{6 2}$ jours après la fin des symptômes. (Le Quotidien du médecin, 16/6/2016)

(27) Une recherche récente suggère que jusqu'à $\mathbf{2 9 \%}$ des bébés de mères infectées développent des problèmes. (Les Échos, 25/7/2016)

Il s'agit dans l'exemple (26) de renvoyer à la transmission par voie sexuelle et à la longue durée d'activité du virus. En (27), c'est le pourcentage de bébés indirectement infectés qui est important. Dans les deux exemples, /jusqu'à $+N u m /$, oriente vers le plus, et par là-même la peur du virus se trouve amplifiée.

Le patron quantifieur /autour de $+\mathrm{Num} /$ fonctionne comme un opérateur d'arrondissement. Faute de données exactes ou par précaution, le journaliste propose une estimation approximative, sans limite inférieure ni supérieure. Cette estimation qui gravite autour d'un chiffre de référence, comme dans (28) est faible de vérification sans pour autant être dénuée d'authenticité :

(28) Il faut y ajouter les patients n'ayant pas consulté, plus la multitude d'asymptomatiques, estimée autour de $\mathbf{8 0 \%}$. (Le Quotidien du médecin, 29/3/2016).

À noter que ce pourcentage « $80 \%$ », lié à l'apparition des symptômes et présenté comme approximatif en (28), est donné comme précis dans d'autres journaux (29, 30 et 31$)$ :

(29) Nous connaissons encore peu le virus Zika dans la mesure où il reste asymptomatique dans $80 \%$ des cas, rappelle le professeur Yazdan Yazdanpanah, chef du service maladies infectieuses et tropicales à l'Hôpital Bichat, à Paris. (L'Express.fr, 5/2/2016)

(30) Mais la recommandation devrait concerner toutes les femmes enceintes puisque dans $\mathbf{8 0 \%}$ des infections, il n'y a pas de symptôme. (Le Point.fr, $3 / 2 / 2016)$

(31) Il y a une incertitude énorme quant aux nombres de personnes infectées puisque $\mathbf{8 0 \%}$ des cas sont asymptomatiques. (Le journal du dimanche $J D D, 28 / 1 / 2016)$

Nous supposons donc une réticence marquante et une prudence chez le journaliste en (28) qui rendent les bornes potentielles très proches de ce chiffre-point de référence sans vouloir le considérer comme précis.

Les schèmes /environ $+\mathrm{Num} /$ et /aux alentours de $+\mathrm{Num} /$ sont les jumeaux identiques de /autour de $+\mathrm{Num} /:$ ils ont les mêmes propriétés. Seul environ apparait dans notre échantillon. Nous avons recensé deux occurrences d'/environ $+\mathrm{Num} /$ : 


$\begin{array}{ccc}\text { Mot } & \text { Occurrences } & \text { Rang } \\ \text { Environ } & 2 & 21\end{array}$

(32) Cette maladie, généralement bénigne, entraine des complications dans environ $1 \%$ des cas. (Le Parisien, 21/8/2015)

(33) Les chercheurs estiment que leur échantillon représente environ 15\% des cas de Zika diagnostiqués au Canada. (Le Quotidien, 6/3/2016).

Dans les exemples (32) et (33), aucune présomption de rigueur particulière. L'approximation est donc explicite. lenviron + Num/ témoigne de l'absence de bornes dans les pourcentages des cas cités de maladies ou d'infections.

Avec /plus de+Num/ et /moins de + Num/, une borne inférieure et une borne supérieure sont posées respectivement : dans le premier cas, le nombre exact (non-dit) est légèrement supérieur au nombre publié, dans le second, il y est inférieur.

\begin{tabular}{|ccc|}
\hline Mots & Occurrences & Rang \\
Plus de & 10 & 11 \\
Moins de & 4 & 22 \\
\hline
\end{tabular}

Avec le quantifieur /plus de $+N u m /$, le nombre que l'on tait est souvent redoutable, puisque celui qui constitue la borne inférieure est déjà important :

(34) En 2013, une épidémie a sévi en Polynésie avec plus de 32000 cas, dont quelques encéphalites et quelques polyradiculonévrites aigües du type Guillain-Barré. (Atlantico, 12/8/2015)

(35) Le Brésil est particulièrement touché avec plus de 1,5 million de personnes infectées. (Sciences et Avenir, 18/7/2016)

(36) Mais on estime qu'il faut vacciner plus de $\mathbf{9 0 \%}$ pour éviter une flambée épidémique. (L'Express, 17/2/2016)

(37) La Floride (sud-est) est actuellement l'État le plus touché, avec plus de 500 infections liées à des voyages et désormais 43 cas de contamination locale, principalement par des moustiques. (Le Quotidien du médecin, 29/8/2016)

(38) Avec plus de 1,5 million de cas au Brésil, et plusieurs milliers ailleurs, dont déjà plus de 40000 cas en Colombie, les chercheurs mettent en garde sur les risques de voir les capacités de soins intensifs dépassés, en particulier en dehors des cités urbaines. (Sciences et Avenir,1/3/2016)

Dans les exemples cités (34 à 38), le nombre exact non-dit étant sur l'axe croissant de gravité, le journaliste, pour rassurer le lecteur, se contente de mentionner un nombre inférieur moins choquant.

Dans les énoncés où /moins de/ est suivi d'un numéral, l'effet pragmatique visé est souvent celui de l'atténuation :

(39) Une méningo-encéphalie se voit dans moins de $1 \%$ des cas de même que de rares hépatites, pancréalites ou myocardites. (Atlantico, 12/8/2015). 
En l'occurrence (39), le chiffre «1» marque déjà une quantité minime. Pour accentuer la rareté du phénomène (rapport entre le zika et les autres anomalies mentionnées dans l'exemple), le journaliste utilise /moins de + Num 1/ afin de montrer que le chiffre non-dit est faible et insignifiant, étant donné qu'il est inférieur à 1 .

Outre le patron /quantifieur + Num/, l'approximation se fait par construction d'intervalle (de $X$ à $Y^{15}$, entre $X$ et $Y$ ) et par oscillation (X à Y) : dans l'intervalle, « le couple de prépositions construit un site configuré comme intervalle dans lequel la cible peut s'inscrire » (Mélis, 2003 :10). Pour l'approximation par oscillation, les deux numéraux se succèdent. Même pour ce qui est des chiffres ronds, en cas d'oscillation, « ils doivent se succéder immédiatement, compte tenu de l'intervalle de modulation. Sont donc admis 20 à 25, 20 à 30 ou 200 à 300, mais non 20 à 35, 20 à 50 et 200 à $1200 »$ (Mélis, $2003: 12$ ).

(40) À ce jour, 48 syndromes congénitaux ont été diagnostiqués aux Antilles et en Guyane ; leur taux serait aux Antilles de 1'ordre de 5 à $\mathbf{1 0} \%$ des femmes enceintes infectées. (Le Figaro, 20/2/2016)

(41) Ce virus a déjà touché 1,5 million de personnes au Brésil, et 3 à 4 millions de cas attendus sur le continent américain. (Le Monde.fr, 2/2/2016).

(42) Dans $\mathbf{7 0}$ à $\mathbf{8 0} \%$ des cas d'infection, le sujet atteint ne développe aucun symptôme (Les Échos, 30/1/2016)

(43) Le SGB (Le syndrôme de Guillain-Barré) prend une forme sévère avec une détresse respiratoire dans $\mathbf{2 0}$ à $\mathbf{3 0} \%$ des cas. (Le Monde.fr, 1/3/2016)

Les chiffres précis sur l'expansion du virus n'étant pas définitifs à l'heure de la diffusion de l'information, une approximation par intervalle comme en (40) ou par oscillation comme en (41), en (42) et en (43), présente une solution à tout journaliste faisant passer l'honnêteté au premier plan au lieu de chercher inlassablement à aguicher le destinataire. Quoique le chiffrage qu'il avance ne soit pas quantitativement précis, il reste informatif : il permet des inférences, plus ou moins correctes, liées à l'intervalle ou à l'oscillation créés par les nombres.

Néanmoins, notons au passage que certains exemples extraits de la presse dérogent ouvertement à cet usage correct de la langue souligné par Mélis. Citons entre autres ceux où la succession des chiffres, dans le cas de l'oscillation, n'est pas respectée (en 44 et 45 ) et ceux où il existe des maladresses quant à l'usage des prépositions (46) :

(44) Les symptômes, quand ils existent, apparaissent trois à douze jours après la piqûre, sous forme d'éruption cutanée avec ou sans fièvre. (Le Monde.fr, 2/2/2016)

(45) La microcéphalie est une malformation rare : aux États-Unis, 2 à 12 cas sont recensés pour 10000 naissances. (Le Monde.fr, 2/2/2016)

(46) Les patients souffrent d'une fièvre, de douleurs articulaires, de conjonctivite ou d'éruptions cutanées, puis tout rentre dans l'ordre entre deux à sept jours. (Libération, 27/1/2016)

$\mathrm{x}$ et $\mathrm{y}$ étant deux adjectifs numéraux. 
Outre les quantifieurs de type prépositionnel, l'approximation opère également via des substantifs à valeur numérale (dizaine, vingtaine, cinquantaine, centaine, millier etc.). Nous y décelons, outre l'approximation, une tendance à créer un effet d'intensification :

(47) Aujourd'hui, une cinquantaine de communes sont considérées comme « envahies » par le moustique tigre. (Sud Ouest, 21/8/2015)

(48) Une quinzaine de laboratoires planchent sur Zika dans le monde. ( $L a$ Lettre de l'Expansion, 6/6/2016)

Dans les exemples précités (47) et (48), il y a une évaluation subjective qui est en rapport avec les représentations sociales. L'effet d'intensification est étroitement lié à nos compétences extralinguistiques : 50 communes touchées par un virus, c'est une grande quantité et 15 laboratoires scientifiques qui étudient le phénomène, c'est un nombre considérable.

Il ressort de ce qui précède que la production des approximations (par quantifieurs + Num, par construction d'intervalle) a souvent pour fonction de tranquilliser au lieu de semer le désarroi alors que l'usage des substantifs à valeur numérale a pour fonction d'intensifier

\subsubsection{Les opérateurs argumentatifs ${ }^{16}$}

Dans la même veine (minimiser, atténuer, affaiblir, etc.), certains opérateurs argumentatifs modifient les données chiffrées. Ducrot définit l'opérateur argumentatif comme suit :

Un morphème $\mathrm{X}$ est un opérateur argumentatif s'il y a au moins une phrase $\mathrm{P}$ telle que l'introduction de $\mathrm{X}$ dans $\mathrm{P}$ produit une phrase $\mathrm{P}$ ', dont le potentiel d'utilisation argumentative est différent de celui de $\mathrm{P}$, cette différence ne pouvant se déduire de la différence entre la valeur informative des énoncés P et P' (Ducrot, 1983 : 9-10).

À peine, seul-e-s et seulement sont des opérateurs argumentatifs au sens de Ducrot ; bien que ne spécifiant pas la quantité désignée d'un point de vue informationnel, ils orientent, lorsqu'ils précédent ou suivent le numéral, vers le moins :

(49) Les deux seuls vrais concurrents sur terre de l'espèce humaine-si l'on excepte l'homme lui-même qui est peut-être son pire ennemi- sont les insectes et les micro-organismes. (Atlantico, 12/8/2015)

(59) Seuls 150 cas de ce syndrome (microcéphalie) avaient été signalés l'an dernier. (L'Express, 30/12/2015)

(51) Elle (la revue scientifique américaine Annals of international Medicine) estime que seuls 37 voyageurs pourraient être contaminés au maximum, en se basant sur des personnes ayant assisté à la coupe du monde $2014 \mathrm{au}$ Brésil, et en étudiant les pays d'où les touristes venaient tout en calculant les risques de transmission du virus. (La Tribune (France), 5/8/2016)

\footnotetext{
A l'inverse du connecteur argumentatif, l'opérateur argumentatif a pour champ d'application un énoncé.
} 
(52) Inutile de s'embijouter, sauf pour la beauté de la chose, avec l'un des multiples bracelets répulsifs, aujourd'hui sur le marché. Leur rayon d'action est trop faible-à peine $20 \mathbf{~ c m}$ - autour du poignet. (Ouest-France, 5/8/2015)

(53) On entend souvent dire que le moustique tigre est facile à reconnaître à coup sûr en raison de ses rayures caractéristiques. Oui, sauf qu'en réalité l'espèce est si petite -à peine $\mathbf{5} \mathbf{~ m m}$ - que cette indication n'est pas d'une grande utilité. (Sciences et Avenir, 28/5/2015)

\subsection{Le tout et le néant}

La valeur numérale dans le pourcentage (100\%) indique la totalité. Dans notre corpus, « $100 \%$ » est utilisé comme un indice de vigilance trop élevée comme en (54) ou (55) tandis que le Zéro, rare en ce contexte d'épidémie, reflète l'espoir de salut, le rêve de zones ou de populations sauvées et indemnes, comme en (56) :

(54) En 3 jours : 100\% des fibroblastes ont été infectés! (Sciences et Avenir, 2/7/2015)

(55) On a retrouvé la présence récente du virus Zika chez 100\% des patients atteints de Guillain-Barré (...). (Sciences et Avenir, 1/3/2016)

(56) Au Brésil, Dilma Rousseff et ses ministres se sont mis au vert ce samedi 13 février. Le mot d'ordre était affiché sur leurs T-shirts : "Zika zéro ", alors que le virus a déjà poussé les autorités à déclencher «l'état d'urgence médicale » fin 2015. (RFI.fr, 14/2/2016)

\subsection{Les chiffres exacts : vérité ou illusion?}

Certains aspects témoignent, peu ou prou, de la crédibilité des chiffres ou en donnent l'illusion de vérité et d'objectivité : le non-arrondissement du chiffre des unités et le maintien des fractions et des nombres décimaux. Lorsque le chiffre avancé n'est pas accompagné de modifieur prépositionnel ni d'opérateur argumentatif, on aimerait bien croire que le nombre donné est exact (c'est effectivement le nombre de malades ou de personnes atteintes, de zones touchées etc.). Toutefois, il parait que ces facteurs ne suffisent pas toujours à convaincre le destinataire à se fier aux chiffres : «l'absence de modifieur de quantifieur révèle l'intention du locuteur de laisser l'interprétation non- spécifiée et donc ouverte à toutes les inférences, ce qui crée l'illusion de précision » (Adler et Asnès, $2013: 20$ ). Raison pour laquelle il arrive que le nombre indiqué soit précédé d'un marqueur de reformulation pour affirmer l'exactitude et initier le lecteur à y croire. Tel est bien le cas de au total + Num :

(57) Au total, 22 zones sont désormais concernées par ce dispositif de lutte contre ce le risque de dissémination de la dengue et du chikungunya mais également du virus Zika. (Le Particulier, 7/9/2015)

(58) Au total, 19 cas suspects sont en cours d'investigation. (Le Quotidien du médecin, 29/3/2016)

(59) Au total, 93,4 millions de personnes pourraient être infectées au cours de cette épidémie, selon une étude parue ce lundi dans la revue spécialisée Nature Microbiology. (Les Echos.fr, 25/7/2016). 
Les exemples montrent que le locuteur procède à une récapitulation sommative pour donner plus de rigueur au chiffre. Ce phénomène rappelle l'usage de l'adverbe « exactement » et de l'adjectif « net(te)s » qui souligne cette volonté inaliénable de défendre les chiffres face à un public sceptique et incrédule.

\section{Conclusion}

Faire parler les chiffres, c'est souvent chercher à produire chez le destinataire cet effet de vérité, d'incontestable, d'irréfutable. Nous sommes partie de corpus que nous avons bien ciblé selon des paramètres de temps, de genres, de thèmes, etc., et ce en fonction de l'objectif de départ que nous nous sommes fixée.

Mettre l'information en chiffres permet de donner, comme nous l'avons remarqué, une épaisseur supplémentaire au phénomène, de confirmer la certitude du diagnostic situationnel.

Sur le plan formel, l'écriture en chiffres des nombres éveille les consciences au danger alors que la transcription en lettres adoucit les données quantitatives chiffrées. Sur le plan sémantico-pragmatique, il y a deux orientations subjectivantes : nous constatons d'un côté la stratégie de l'homme de paille, sorte d'épouvantail qui cherche à terrifier en amplifiant les chiffres pour que les populations soient sur leurs gardes. Il est question d'un appel à la peur rendu efficace grâce à l'exposition de la sévérité de la menace par les chiffres. De l'autre côté, il y a la stratégie d'apaisement du type « tout est bien, la situation est sous contrôle » qui est incarné par les éléments modificateurs (quantifieurs et opérateurs) qui, comme nous l'avons vu, gomment la peur en minimisant le danger. L'écart entre le niveau factuel ou informatif d'une part, et le niveau argumentatif et le point de vue du journaliste d'autre part n'est souvent que le reflet du passage des résultats numériques officiels, témoins de la vérité, à des ersatz subjectifs de la réalité.

\section{Références bibliographiques :}

Adler, S. et Asnès, M., (2004) «Les compléments de degré en jusqu'à » in Travaux de linguistique. 2004/2 n²9, pp. 133-159.

Adler, S. et Asnès, M., (2007) «Le monde d'au-delà : une dimension scalaire ». Travaux de linguistique, Vol. 1, n 54 , pp. 29-42.

Adler, S. et Asnès, M., (2008) «Approximation par arrondissement : le cas de quelques quantifieurs prépositionnels ", in Actes du Congrès Mondial de la linguistique française (CMLF'2008) Paris, CNRS et Institut de linguistique française, Disponible sur : http:// dx.doi.org/10.10.1051/cmlf08084

Adler, S. et Asnès, M., (2010) «Autour de la précision ». In l'Information grammaticale ${ }^{\circ}$ 125, pp. 36-43.

Adler, S. et Asnès, M., (2013) «Qui sème la quantification récolte l'intensification ». Langue française, Vol. 1, n 177 , pp. 9-22.

Condamines, A. et Dehaut, N., (2011) « Mise en œuvre des méthodes de la linguistique de corpus pour étudier les termes en situation d'innovation disciplinaire : le cas de l'exobiologie », in Meta 562, pp. 266-283 
Doury, M. et Moirand, S., (2004) L'argumentation aujourd'hui. Positions théoriques en confrontation. Paris, Presses de la Sorbonne Nouvelle.

Ducrot, O., (1983) « Opérateurs argumentatifs et visés argumentatives ». In Cahiers de linguistique française, $\mathrm{n}^{\circ} 5$, pp. 7-36.

Ducrot, O., (1995) « Les modificateurs déréaliseants ». In Journal of Pragmatics, n²4, pp. $145-165$.

Ducrot, O., (2002) «Quand peu et un peu semblent co-orientés ». In Cahiers de linguistique française, $\mathrm{n}^{\circ} 24$, pp. 207-229.

Hylande, K., (2009) Academic discourse. London, Continuum.

Jacobi, D. et Schiele, B., (éds) (1988). Vulgariser la Science. Seyssel, éd. Champs Vallon.

Soutet, O., (2010) «Genèse et sens de quelque : essai de microsémantique historique ». In L'information grammaticale, $\mathrm{n}^{\circ} 125, \mathrm{pp} .22-28$.

Melis, L., (2003) «Les quantificateurs approximatifs du type prépositionnel ». In Verbum $\mathrm{n}^{\circ} 25,5-24$.

Vanneuville, R., (2012) «Le chiffre au service du droit ou le droit au service du chiffre ? ». iIn Mots. Les langages du politique [En ligne], 2013s, 123-136 consulté le 20 mars 2013. Disponible sur : http:// mots.revues.org/20985

Vauger, C., (2005) « Dans les + Numéral : un déterminant de quantification faible ? ». In Travaux de linguistique Vol. 1 n50, De Boeck supérieur, pp. 113-129. 